\title{
ЭЛЕКТРОДИНАМИЧЕСКИЙ РАСЧЕТ ЭФФЕКТИВНЫХ ЭЛЕКТРОМАГНИТНЫХ ПАРАМЕТРОВ ДИЭЛЕКТРИЧЕСКОЙ СРЕДЫ С МЕТАЛЛИЧЕСКИМИ НАНОЧАСТИЦАМИ ЗАДАННЫХ РАЗМЕРОВ
}

\author{
Б. А. Беляев ${ }^{a, b, c^{*}}$, В. В. Тюрнев ${ }^{a, b}$ \\ ${ }^{a}$ Институт физики им. Л. В. Киренского ФИЦ КНЦ Сибирского отделения Российской академии наук \\ 660036, Красноярск, Россия \\ ${ }^{b}$ Сибирский федералъный университет \\ 660041, Красноярск, Россия \\ ${ }^{c}$ Сибирский государственный университет науки и технологий им. М. Ф. Решетнева \\ 660037, Красноярск, Россия
}

Поступила в редакцию 22 марта 2018 г.

\begin{abstract}
Оригинальным методом рассчитаны частотные зависимости эффективной комплексной диэлектрической проницаемости и эффективной комплексной магнитной восприимчивости гетероструктуры на основе диэлектрической среды, содержащей металлические наночастицы сферической формы. В отличие

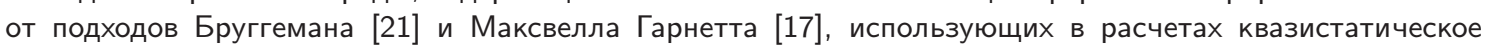
приближение, рассчитано неоднородное распределение электромагнитных полей внутри металлических наночастиц, что позволило исследовать зависимость электромагнитных параметров гетероструктуры не только от частоты, но и от размера частиц. Показано, что частота плазмонного резонанса понижается с увеличением как размера частиц, так и их концентрации в гетероструктуре. Показано также, что диэлектрическая среда, содержащая немагнитные металлические наночастицы, проявляет диамагнитные свойства. При этом положение максимума на частотной зависимости мнимой части магнитной восприимчивости совпадает с частотой релаксации носителей заряда. Рассчитанные спектры действительной и мнимой компонент диэлектрической проницаемости гетероструктуры с размерами металлических частиц меньше 10 нм хорошо согласуются с расчетами Бруггемана, однако с расчетами Максвелла Гарнетта согласие наблюдается только при концентрациях наночастиц меньше $10^{-6}$.
\end{abstract}

\section{DOI: $10.1134 / \mathrm{S} 00444 \overline{1} 1018 \overline{10} 0036$}

\section{1. ВВЕДЕНИЕ}

Современные технологии позволяют не только изготавливать металлические наночастицы сферической формы размерами от единиц до сотен нанометров, но и создавать на их основе взвеси в водной среде (суспензии различных концентраций), исполь-

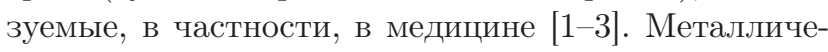
ские наночастицы используются также для усиления электромагнитного поля вблизи длинных молекулярных цепочек красителей, что приводит к образованию локальной бистабильности в окрестности частицы [īin]. Хорошо известно, что в металли-

\footnotetext{
* E-mail: belyaev@iph.krasn.ru
}

ческих наночастицах наблюдаются плазмонные резонансы, частоты которых попадают в оптический диапазон [1; основе диэлектрической среды, содержащей металлические наночастицы, включая водные суспензии, имеют цветовую окраску в зависимости от размеров наночастиц [푝. С учетом того, что плазмонные резонансы в частицах должны проявляться на частотных зависимостях электромагнитных параметров гетероструктур, задача расчета эффективных комплексных диэлектрической и магнитной проницаемостей в них представляет большой интерес и является актуальной.

Известно, что вынужденные электромагнитные колебания в любом однородном теле сферической формы под воздействием падающей на него снару- 
жи плоской электромагнитной волны представляют собой суперпозицию электрических и магнитных мультипольных колебаний от первого порядка до бесконечности. Компоненты электрического и магнитного полей таких колебаний описываются сферическими функцияи [10]. Известно также, что резонансы мультипольных электрических и магнитных колебаний в диэлектрических сферах наблюдаются на частотах, при которых размеры сферы соизмеримы с длиной электромагнитной волны в диэлектрическом материале. Однако для электрических мультипольных колебаний в металлических наночастицах, которые часто называют плазмонными колебаниями, наблюдаются резонансные возбуждения (плазмоны) на частотах, когда длина волны $\lambda_{m}$ в материале много больше радиуса $a$ частицы [[-6]. Плазмоны различной мультипольности влияют на характер рассеяния электромагнитных волн на металлических наночастицах, расположенных в диэлектрической матрице [1] 1iㄹ.

Резонансные частоты $\omega_{n}$ электрических мультипольных колебаний определяются уравнениями

$$
(n+1) \varepsilon_{d}+n \varepsilon_{m}(\omega)=0 .
$$

Эти уравнения получаются из условия обращения в нуль знаменателей амплитуд электрических полей [1] при $a \rightarrow 0$. Здесь $\varepsilon_{d}-$ относительная диэлектрическая проницаемость окружающей среды, a $\varepsilon_{m}(\omega)$ - относительная комплексная диэлектрическая проницаемость материала наночастицы, которая в рамках модифицированной модели Друде [12 12$]$ выражается формулой

$$
\varepsilon_{m}(\omega)=\varepsilon_{\infty}-\frac{\omega_{p}^{2}}{\omega^{2}+i \omega \gamma},
$$

где $\varepsilon_{\infty}$ - оптическая диэлектрическая проницаемость металла, $\omega_{p}$ - частота плазменных колебаний в металле, а $\gamma$ - частота релаксации носителей заряда. При выводе этой формулы предполагалось, что гармонические колебания описываются множителем $\exp (-i \omega t)$. Из формул $\left(\begin{array}{l}\overline{1} \\ 1\end{array}\right)$ и $(\overline{1})$ (1) резонансные частоты $\omega_{n}$ и добротности $Q_{n}$ электрических мультипольных колебаний при $2 \pi a \ll \lambda_{m}$ выражаются формулами

$$
\begin{aligned}
\omega_{n} & =\sqrt{\frac{\omega_{p}^{2}}{\left(1+n^{-1}\right) \varepsilon_{d}+\varepsilon_{\infty}}-\left(\frac{\gamma}{2}\right)^{2}}, \\
Q_{n} & =\sqrt{\frac{\omega_{p}^{2} \gamma^{-2}}{\left(1+n^{-1}\right) \varepsilon_{d}+\varepsilon_{\infty}}-\frac{1}{4}}
\end{aligned}
$$

Самую большую амплитуду имеют плазмонные колебания с $n=1$, т. е. электродипольные колебания.
Амплитуды других плазмонных колебаний (квадрупольных, октупольных и т. д.) быстро убывают как с увеличением их порядка $n$, так и с уменьшением радиуса $а$ частицы.

Отметим, что вынужденные магнитные мультипольные колебания в сферических металлических наночастицах не имеют резонансов в области оптических частот, так как вещественная часть знаменателей их амплитуд не обращается в нуль при $a<\lambda_{m}$ [1]3]. Однако в присутствии постоянного магнитного поля магнитодипольный резонанс наблюдался наряду с электрическим дипольным резонансом [11 4,

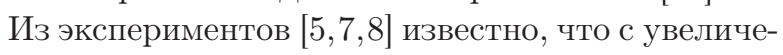
нием радиуса $a$ частиц частота $\omega_{1}$ плазмонных колебаний металлической наночастицы начинает плавно понижаться, но только когда радиус $а$ превыша-

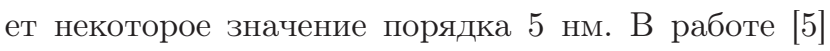
этот эффект объясняли наличием нескольких механизмов затухания плазмонов, используя квазистатическое приближение. В другой работе [1 $\left.15 \overline{5}_{1}^{\prime}\right]$ зависимость резонансной частоты плазмонных колебаний от размера металлической наночастицы объяснялось уже квантовыми размерно-зависимыми эффектами и также в квазистатическом приближении. Однако, как показано в работе ["⿺辶一]|, зависимости резонансных частот мультипольных плазмонных колебаний от размера частицы можно получить электродинамическим расчетом распределения электромагнитных полей внутри частицы, т.е. отказаться от использования квазистатического приближения, в котором считается, что локальные высокочастотные свойства материала наночастицы однородны по всему ее объему и совпадают с локальными свойствами массивного материала.

Для расчета эффективной комплексной диэлектрической проницаемости $\varepsilon_{e f f}$ диэлектрической среды, содержащей металлические наночастицы, большинство авторов также используют электродипольное квазистатическое приближение. Поэтому полученные ими формулы не содержат радиуса $a$ металлических наночастиц, а значит, не описывают влияния размера наночастиц на частотную дисперсию $\varepsilon_{e f f}(\omega)$ вблизи частоты $\omega_{1}$ плазмонного резонанса. $\mathrm{K}$ числу таких формул относится формула Максвелла-Вагнера [1] $\left.{ }_{1}^{\prime}\right]$

$$
\varepsilon_{e f f}=\varepsilon_{d}\left[1+3 c_{m} \frac{\varepsilon_{m}-\varepsilon_{d}}{\varepsilon_{m}+2 \varepsilon_{d}} /\left(1-c_{m} \frac{\varepsilon_{m}-\varepsilon_{d}}{\varepsilon_{m}+2 \varepsilon_{d}}\right)\right]
$$

и идентичная ей формула Максвелла Гарнет-

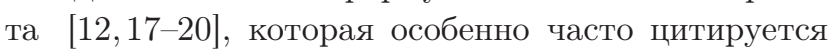
исследователями, а также формула Бруггемана $\left[20_{1}^{\prime}, 21_{1}^{\prime}\right]$ 


$$
\begin{gathered}
\varepsilon_{e f f}=\frac{H_{B}+\sqrt{H_{B}^{2}+8 \varepsilon_{m} \varepsilon_{d}}}{4}, \\
H_{B}=\left(2-3 c_{m}\right) \varepsilon_{d}-\left(1-3 c_{m}\right) \varepsilon_{m} .
\end{gathered}
$$

Здесь $c_{m}$ обозначает объемную концентрацию металлических наночастиц в диэлектрической среде.

Формула (푼) получена в приближении малой концентрации $c_{m}$ металлических наночастиц в гетероструктуре, когда взаимодействием частиц можно пренебречь. В формуле $\left(\overline{6}_{6}^{+}\right)$взаимодействие между наночастицами учтено только в приближении среднего поля. Значения $\varepsilon_{e f f}$, рассчитанные по этим двум формулам, совпадают только при очень малых концентрациях $\left(c_{m}<1 \cdot 10^{-6}\right)$, однако уже при $c_{m}=1 \cdot 10^{-5}$ эти значения сильно различаются. При этом из обеих формул следует, что резонансная частота $\omega_{1}$ дипольных плазмонных колебаний выражается формулой $(\overline{3})$ при $n=1$.

В работе [22] проведено сравнение существующих вариантов уточнения теории Максвелла Гарнетта для учета влияния размера наночастиц на $\varepsilon_{e f f}(\omega)$. В одном из вариантов [1현] было предложено статическую электрическую поляризуемость наночастицы заменить на электродинамическую поляризуемость, которая описывается формулами теории Ми ["ín.". Такой же подход был использован и в работе [23] при выводе формулы для расчета эффективной магнитной проницаемости $\mu_{e f f}$ гетероструктуры.

В работе [24'] для учета зависимости эффективной проницаемости от размера частиц используется формализм интегральных уравнений и функций Грина. Однако такой формализм предполагает однородность электрического поля внутри наночастицы, что является грубым приближением, но оно используется и в работах $[25,256]$.

Таким образом, известные формулы для расчета эффективной комплексной диэлектрической проницаемости, позволяющие учесть зависимость $\varepsilon_{e f f}$ от радиуса $a$ металлических наночастиц в гетероструктуре, получены на основе подхода Максвелла Гарнетта. Однако значительное расхождение даже при низкой концентрации наночастиц в гетероструктуpe $\left(c_{m}=1 \cdot 10^{-5}\right)$ между результатами расчета $\varepsilon_{e f f}$ по квазистатической формуле Максвелла Гарнетта и по квазистатической формуле Бруггемана показывает, что при выводе этих формул допущены слишком грубые приближения.

Мотивацией проведенных в настоящей работе исследований является необходимость разработки модели и проведения расчета гетероструктуры, состоящей из диэлектрической матрицы с металлически- ми наночастицами, для более точного описания диэлектрических свойств изучаемого объекта по сравнению с моделями Максвелла Гарнетта и Бруггемана. Амплитуды электромагнитных полей в разработанной модели считаются неоднородными в объеме частицы, что с физической точки зрения является абсолютно правильным в условиях плазмонных возбуждений в металлических частицах. Другими словами, частицы бесконечно малых размеров заменены частицами с реальными размерами. Взаимодействие частиц учитывается с использованием подхода Бруггемана. В результате получены формулы для расчета эффективной комплексной диэлектрической проницаемости, а также эффективной магнитной проницаемости рассматриваемой гетероструктуры, что позволило провести исследования зависимости электромагнитных параметров среды от частоты, от размеров наночастиц и от их концентрации в гетероструктуре. Правильность разработанного подхода доказывает, в частности, существенное понижение частоты плазмонного резонанса с увеличением размера металлических частиц, наблюдаемое экспериментально в водных суспензи-

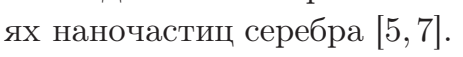

\section{2. СРАВНЕНИЕ ПОДХОДОВ МАКСВЕЛЛА ГАРНЕТТА И БРУГГЕМАНА ПРИ РАСЧЕТЕ ЭФФЕКТИВНЫХ ПАРАМЕТРОВ ГЕТЕРОСТРУКТУР}

В основе подхода Максвелла Гарнетта лежит приближенная формула

$$
\varepsilon_{e f f}=\varepsilon_{d}+\frac{P}{\varepsilon_{0} E}
$$

где $\varepsilon_{0}-$ абсолютная диэлектрическая проницаемость вакуума, $\varepsilon_{e f f}$ - относительная эффективная диэлектрическая проницаемость гетероструктуры с наночастицами, $\varepsilon_{d}$ - относительная диэлектрическая проницаемость матрицы гетероструктуры, $P$ диэлектрическая поляризация металлических наночастиц под воздействием электромагнитной волны с амплитудой электрического поля $E$. В этом подходе

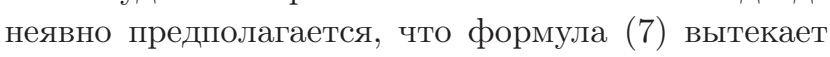
из известной формулы

$$
D \equiv \varepsilon_{0} E+P=\varepsilon_{0} \varepsilon_{d} E,
$$

используемой для описания диэлектрических свойств только сплошных однородных сред, в которых не могут возбуждаться никакие мультипольные колебания, кроме дипольных. Это значит, что такой 
подход принципиально не позволяет описать хорошо наблюдаемые экспериментально мультипольные возбуждения высших порядков в металлических наночастицах.

Кроме того, серьезная погрешность в подходе Максвелла Гарнетта заключается в том, что в расчете дипольные моменты реальных сферических металлических частиц радиуса а заменяются на эквивалентные моменты диполей, помещенных в центры частиц, уменьшенных до точечных размеров ( $a=$ $=0)$. При такой замене реальное электрическое поле в наночастице имеет сингулярность. Однако эта сингулярность поля не принимается во внимание и считается, что поле внутри частицы такое же, как и в окружающей матрице. В результате исключается деполяризующее поле внутри частицы, а поэтому преувеличивается вклад металлических частиц в эффективную диэлектрическую проницаемость гетероструктуры.

В основе подхода Бруггемана лежит строгая формула, отражающая закон непрерывности потока индукции электрического поля:

$$
\Delta \Phi \equiv \iint \varepsilon_{r}(\mathbf{r}) E_{n}(\mathbf{r}) d s-\varepsilon_{e f f} \iint E_{0} d s=0,
$$

где $\Delta \Phi-$ скачок потока индукции электрического поля на поверхности интегрирования, $E_{n}(\mathbf{r})-$ нормальная к поверхности интегрирования компонента микроскопического электрического поля, $\varepsilon_{r}(\mathbf{r})-$ локальная относительная диэлектрическая проницаемость гетероструктуры, принимающая значение $\varepsilon_{m}$ внутри выделенной металлической наночастицы, $\varepsilon_{d}$ внутри выделенной диэлектрической частицы и значение $\varepsilon_{e f f}$ вне выделенной частицы, $\mathbf{E}_{0}-$ макроскопическое электрическое поле волны, ортогональное поверхности интегрирования. Эта формула получается интегрированием уравнения Максвелла

$$
\operatorname{div}\left(\varepsilon_{r} \mathbf{E}\right)=0
$$

по любой поверхности, ортогональной макроскопическому полю $\mathbf{E}_{0}$. Очевидно, что эта поверхность является поверхностью усреднения микроскопического поля. Так как гетероструктура считается макроскопически однородной, место расположения для поверхности усреднения микроскопического поля может быть выбрано произвольно. Очевидно, что понятие эффективная диэлектрическая проницаемость $\varepsilon_{e f f}$ имеет смысл только в случае, когда поперечные размеры поверхности усреднения больше поперечного размера наночастицы, но много меньше длины волны в гетероструктуре.
Заметим, что в подходе Бруггемана в явном виде рассматривается только одна наночастица, однако ее взаимодействие с другими наночастицами все же учитывается, но только в приближении усредненного поля в гетероструктуре, характеризующейся параметром $\varepsilon_{e f f}$.

Таким образом, при расчете эффективной комплексной диэлектрической проницаемости диэлектрической среды с металлическими наночастицами подход Бруггемана является более строгим, чем подход Максвелла Гарнетта.

\section{3. РАСЧЕТ ЭФФЕКТИВНЫХ ЭЛЕКТРОМАГНИТНЫХ ПАРАМЕТРОВ ДИЭЛЕКТРИЧЕСКОЙ СРЕДЫ С МЕТАЛЛИЧЕСКИМИ НАНОЧАСТИЦАМИ}

Будем считать, что рассматриваемая гетероструктура является средой, состоящей из диэлектрических и металлических наношариков. Интегральный скачок потока индукции электрического поля, $\Delta \Phi$, на поверхности интегрирования, вычисляемый по формуле (宓), представим в виде суммы скачков от одиночных наночастиц в усредненной среде:

$$
\Delta \Phi=n_{m} \Delta \Phi_{m}+n_{d} \Delta \Phi_{d}
$$

где $n_{m}$ и $n_{d}$ - число наночастиц соответственно металла и диэлектрика в области интегрирования, а $\Delta \Phi_{m}$ и $\Delta \Phi_{d}$ - их вклады от отдельной наночастицы, находящейся в однородной среде с параметрами $\varepsilon_{\text {eff }}$ и $\mu_{\text {eff }}$.

Начнем расчет со скачка $\Delta \Phi_{m}$. Для этого рассмотрим распространение плоской электромагнитной волны в однородной среде, содержащей одиночную сферическую металлическую наночастицу. Пусть компоненты макроскопического поля волны выражаются формулами

$$
\begin{aligned}
& E_{x}(z)=E_{0} \exp \left(i k_{e f f} z\right), \\
& H_{y}(z)=H_{0} \exp \left(i k_{e f f} z\right),
\end{aligned}
$$

где амплитуды электрического и магнитного полей связаны соотношением $E_{0}=Z_{e f f} H_{0}$, а волновое число $k_{e f f}$ и волновое сопротивление $Z_{e f f}$ заданы формулами

$$
k_{e f f}=\sqrt{\varepsilon_{e f f} \mu_{e f f}} \frac{\omega}{c},
$$

$$
Z_{e f f}=Z_{0} \sqrt{\frac{\mu_{e f f}}{\varepsilon_{e f f}}}, \quad Z_{0}=\sqrt{\frac{\mu_{0}}{\varepsilon_{0}}} .
$$


Выделим в гетероструктуре одну наночастицу, и в ее центр поместим точку начала отсчета координат. Рассмотрим микроскопическое электромагнитное поле внутри и вблизи этой наночастицы. Снаружи оно складывается из поля падающей волны, заданной формулой $\left(\overline{1} \overline{1}_{1}^{\prime}\right)$, и поля отраженной волны. Внутри частицы это поле характеризуется полем прошедшей волны. Как известно ты поля отраженной волны выражаются формулами

$$
\begin{aligned}
& \mathbf{E}^{r}=E_{0} \sum_{n=1}^{\infty} i^{n} \frac{2 n+1}{n(n+1)}\left(A_{n}^{r} \mathbf{m}_{o 1 n}^{r}-i B_{n}^{r} \mathbf{n}_{e 1 n}^{r}\right), \\
& \mathbf{H}^{r}=H_{0} \sum_{n=1}^{\infty} i^{n} \frac{2 n+1}{n(n+1)}\left(-i A_{n}^{r} \mathbf{n}_{o 1 n}^{r}-B_{n}^{r} \mathbf{m}_{e 1 n}^{r}\right),
\end{aligned}
$$

где векторные функции $\mathbf{m}_{o 1 n}^{r}(r, \theta, \varphi), \mathbf{n}_{o 1 n}^{r}(r, \theta, \varphi)$, $\mathbf{m}_{e 1 n}^{r}(r, \theta, \varphi), \mathbf{n}_{e 1 n}^{r}(r, \theta, \varphi)$ определены в сферической системе координат формулами

$$
\begin{aligned}
& \mathbf{m}_{e^{1 n}}^{r}=-\sin \varphi h_{n}^{(1)}\left(k_{e f f} r\right) \frac{P_{n}^{1}(\cos \theta)}{\sin \theta} \mathbf{i}_{\theta}- \\
& -{ }_{\cos }^{\sin } \varphi h_{n}^{(1)}\left(k_{e f f} r\right) \frac{d P_{n}^{1}(\cos \theta)}{d \theta} \mathbf{i}_{\varphi}, \\
& \mathbf{n}_{e^{o}{ }_{1 n}^{r}}^{r}={ }_{\cos }^{\sin } \varphi n(n+1) \frac{h_{n}^{(1)}\left(k_{e f f} r\right)}{k_{e f f} r} \times \\
& \times P_{n}^{1}(\cos \theta) \mathbf{i}_{r}+\sin _{\cos } \varphi \frac{\left[k_{e f f} r h_{n}^{(1)}\left(k_{e f f} r\right)\right]^{\prime}}{k_{e f f} r} \times \\
& \times \frac{d P_{n}^{1}(\cos \theta)}{d \theta} \mathbf{i}_{\theta}+\underset{-\sin }{\cos } \varphi \times \\
& \times \frac{\left[k_{e f f} r h_{n}^{(1)}\left(k_{e f f} r\right)\right]^{\prime}}{k_{e f f} r} \frac{P_{n}^{1}(\cos \theta)}{\sin \theta} \mathbf{i}_{\varphi},
\end{aligned}
$$

а коэффициенты $A_{n}^{r}$ и $B_{n}^{r}$, являющиеся амплитудами соответственно магнитных и электрических мультипольных возбуждений $n$-го порядка, определены формулами

$$
\begin{aligned}
& A_{n}^{r}=-\frac{\mu_{m} j_{n}\left(k_{m} a\right)\left[k_{e f f} a j_{n}\left(k_{e f f} a\right)\right]^{\prime}-\mu_{e f f} j_{n}\left(k_{e f f} a\right)\left[k_{m} a j_{n}\left(k_{m} a\right)\right]^{\prime}}{\mu_{m} j_{n}\left(k_{m} a\right)\left[k_{e f f} a h_{n}^{(1)}\left(k_{e f f} a\right)\right]^{\prime}-\mu_{e f f} h_{n}^{(1)}\left(k_{e f f} a\right)\left[k_{m} a j_{n}\left(k_{m} a\right)\right]^{\prime}}, \\
& B_{n}^{r}=-\frac{\varepsilon_{m} j_{n}\left(k_{m} a\right)\left[k_{e f f} a j_{n}\left(k_{e f f} a\right)\right]^{\prime}-\varepsilon_{e f f} j_{n}\left(k_{e f f} a\right)\left[k_{m} a j_{n}\left(k_{m} a\right)\right]^{\prime}}{\varepsilon_{m} j_{n}\left(k_{m} a\right)\left[k_{e f f} a h_{n}^{(1)}\left(k_{e f f} a\right)\right]^{\prime}-\varepsilon_{e f f} h_{n}^{(1)}\left(k_{e f f} a\right)\left[k_{m} a j_{n}\left(k_{m} a\right)\right]^{\prime}} .
\end{aligned}
$$

Здесь $j_{n}(x)$ и $h_{n}^{(1)}(x)$ - сферические функции Бесселя, порядок $n$ которых совпадает с порядком мультипольного возбуждения, $P_{n}^{m}(x)-$ присоединенная функция Лежандра первого рода, $\mu_{m}$ и $\mu_{e f f}-$ соответственно относительная магнитная проницаемость металла наночастицы и относительная эффективная магнитная проницаемость гетероструктуры, а волновое число для металла наночастицы определяется формулой

$$
k_{m}=\sqrt{\varepsilon_{m} \mu_{m}} \omega / c .
$$

Знак штрих над квадратной скобкой обозначает дифференцирование по $k_{m} a$ или по $k_{e f f} a$ в зависимости от содержимого скобки.

Компоненты поля волны, прошедшей в наночастицу, выражаются формулами

$$
\begin{aligned}
\mathbf{E}^{t}=E_{0} \sum_{n=1}^{\infty} i^{n} \frac{2 n+1}{n(n+1)}\left(A_{n}^{t} \mathbf{m}_{o 1 n}^{t}-i B_{n}^{t} \mathbf{n}_{e 1 n}^{t}\right), \\
\mathbf{H}^{t}=\frac{Z_{e f f}}{Z_{m}} H_{0} \sum_{n=1}^{\infty} i^{n} \frac{2 n+1}{n(n+1)} \times \\
\times\left(-i A_{n}^{t} \mathbf{n}_{o 1 n}^{t}-B_{n}^{t} \mathbf{m}_{e 1 n}^{t}\right),
\end{aligned}
$$

где $Z_{m}$ - волновое сопротивление металлической среды наночастицы,

$$
Z_{m}=Z_{0} \sqrt{\mu_{m} / \varepsilon_{m}}
$$

векторные функции $\mathbf{m}_{o 1 n}^{t}(r, \theta, \varphi), \quad \mathbf{n}_{o 1 n}^{t}(r, \theta, \varphi)$, $\mathbf{m}_{e 1 n}^{t}(r, \theta, \varphi), \mathbf{n}_{e 1 n}^{t}(r, \theta, \varphi)$ определены формулами

$$
\begin{aligned}
& \mathbf{m}_{{ }_{e}{ }_{1 n}^{t}}^{t}={ }_{-\sin }^{\cos } \varphi j_{n}\left(k_{m} r\right) \frac{P_{n}^{1}(\cos \theta)}{\sin \theta} \mathbf{i}_{\theta}- \\
& -{ }_{\cos }^{\sin } \varphi j_{n}\left(k_{m} r\right) \frac{d P_{n}^{1}(\cos \theta)}{d \theta} \mathbf{i}_{\varphi}, \\
& \mathbf{n}_{e^{1 n}}^{t}={ }_{\cos }^{\sin } \varphi n(n+1) \frac{j_{n}\left(k_{m} r\right)}{k_{m} r} \times \\
& \times P_{n}^{1}(\cos \theta) \mathbf{i}_{r}+\sin _{\cos } \varphi \frac{\left[k_{m} r j_{n}\left(k_{m} r\right)\right]^{\prime}}{k_{m} r} \times \\
& \times \frac{d P_{n}^{1}(\cos \theta)}{d \theta} \mathbf{i}_{\theta}+\underset{-\sin }{\cos } \varphi \times \\
& \times \frac{\left[k_{m} r j_{n}\left(k_{m} r\right)\right]^{\prime}}{k_{m} r} \frac{P_{n}^{1}(\cos \theta)}{\sin \theta} \mathbf{i}_{\varphi},
\end{aligned}
$$

а амплитуды задаются формулами 


$$
\begin{aligned}
& A_{n}^{t}=\frac{\mu_{m} j_{n}\left(k_{e f f} a\right)\left[k_{e f f} a h_{n}^{(1)}\left(k_{e f f} a\right)\right]^{\prime}-\mu_{m} h_{n}^{(1)}\left(k_{e f f} a\right)\left[k_{e f f} a j_{n}\left(k_{e f f} a\right)\right]^{\prime}}{\mu_{m} j_{n}\left(k_{m} a\right)\left[k_{e f f} a h_{n}^{(1)}\left(k_{e f f} a\right)\right]^{\prime}-\mu_{e f f} h_{n}^{(1)}\left(k_{e f f} a\right)\left[k_{m} a j_{n}\left(k_{m} a\right)\right]^{\prime}}, \\
& B_{n}^{t}=\frac{\varepsilon_{m} j_{n}\left(k_{e f f} a\right)\left[k_{e f f} a h_{n}^{(1)}\left(k_{e f f} a\right)\right]^{\prime}-\varepsilon_{m} h_{n}^{(1)}\left(k_{e f f} a\right)\left[k_{e f f} a j_{n}\left(k_{e f f} a\right)\right]^{\prime}}{\varepsilon_{m} j_{n}\left(k_{m} a\right)\left[k_{e f f} a h_{n}^{(1)}\left(k_{e f f} a\right)\right]^{\prime}-\varepsilon_{e f f} h_{n}^{(1)}\left(k_{e f f} a\right)\left[k_{m} a j_{n}\left(k_{m} a\right)\right]^{\prime}} \frac{Z_{m}}{Z_{e f f}} .
\end{aligned}
$$

Подробнее рассмотрим электромагнитное поле в двух ортогональных плоскостях, проходящих через центр наночастицы и ортогональных векторам $\mathbf{E}_{0}$ и $\mathbf{H}_{0}$ падающей волны. Заметим, что этим плоскостям ортогонален только единственный орт $\mathbf{i}_{\varphi}$ сферической системы координат. Из формул (11 $\left.\overline{1}_{1}^{\prime}\right)$ и (1) получаем ортогональные компоненты поля в декартовой системе координат:

$$
\begin{aligned}
E_{x}^{r}=E_{0} \sum_{n=1}^{\infty} i^{n} \frac{2 n+1}{n(n+1)} \times & \left(A_{n}^{r} h_{n}^{(1)}\left(k_{e f f} r\right) \frac{d P_{n}^{1}(\cos \theta)}{d \theta}-\right. \\
& \left.-i B_{n}^{r} \frac{\left[k_{e f f} r h_{n}^{(1)}\left(k_{e f f} r\right)\right]^{\prime}}{k_{e f f} r} \frac{P_{n}^{1}(\cos \theta)}{\sin \theta}\right), \\
H_{y}^{r}=H_{0} \sum_{n=1}^{\infty} i^{n} \frac{2 n+1}{n(n+1)} \times & \times\left(-i A_{n}^{r} \frac{\left[k_{e f f} r h_{n}^{(1)}\left(k_{e f f} r\right)\right]^{\prime}}{k_{e f f} r} \frac{P_{n}^{1}(\cos \theta)}{\sin \theta}+\right. \\
\times & \left.+B_{n}^{r} h_{n}^{(1)}\left(k_{e f f} r\right) \frac{d P_{n}^{1}(\cos \theta)}{d \theta}\right),
\end{aligned}
$$

и

$$
\begin{aligned}
E_{x}^{t}= & E_{0} \sum_{n=1}^{\infty} i^{n} \frac{2 n+1}{n(n+1)} \times \\
& \times\left(A_{n}^{t} j_{n}\left(k_{m} r\right) \frac{d P_{n}^{1}(\cos \theta)}{d \theta}-\right. \\
& \left.-i B_{n}^{t} \frac{\left[k_{m} r j_{n}\left(k_{m} r\right)\right]^{\prime}}{k_{m} r} \frac{P_{n}^{1}(\cos \theta)}{\sin \theta}\right), \\
H_{y}^{t}= & \frac{Z_{e f f}}{Z_{m}} H_{0} \sum_{n=1}^{\infty} i^{n} \frac{2 n+1}{n(n+1)} \times \\
\times & \left(-i A_{n}^{t} \frac{\left[k_{m} r j_{n}\left(k_{m} r\right)\right]^{\prime}}{k_{m} r} \frac{P_{n}^{1}(\cos \theta)}{\sin \theta}+\right. \\
+B_{n}^{t} j_{n}\left(k_{m} r\right) & \left.\frac{d P_{n}^{1}(\cos \theta)}{d \theta}\right) .
\end{aligned}
$$

Согласно формулам (害) и $\left(\mathbf{1} \mathbf{1}_{0}^{\prime}\right)$, скачок $\Delta \Phi_{m}$ выражается в сферической системе координат формулой

3 ЖЭТФ, вып. 4 (10)

$$
\begin{array}{r}
\Delta \Phi_{m}=2 \int_{\theta=0}^{\pi} \int_{r=0}^{a}\left(\varepsilon_{m} E_{x}^{1}-\varepsilon_{e f f} E_{0}\right) r d r d \theta+ \\
+2 \varepsilon_{e f f} \int_{\theta=0}^{\pi} \int_{r=0}^{R} E_{x}^{r} r d r d \theta,
\end{array}
$$

где $R$ - радиус области на плоскости $x=0$, по которой проводится усреднение микроскопического поля.

Прежде чем подставить формулы (둔) и $(2 \overline{2}-1)$ в уравнение (25) и проинтегрировать его, выполним сначала интегрирование компонент $E_{x}^{r}$ и $E_{x}^{t}$ только по углу $\theta$, после чего в интегралах исчезает вклад от некоторой части мультипольных возбуждений, а получающиеся выражения становятся более простыми:

$$
\begin{gathered}
\int_{\theta=0}^{\pi} E_{x}^{r} d \theta=E_{0} \sum_{n=1,3,5, \ldots}^{\infty} i^{n-1} \frac{2 n+1}{n(n+1)} \times \\
\times B_{n}^{r} \frac{\left[k_{e f f} r h_{n}^{(1)}\left(k_{e f f} r\right)\right]^{\prime}}{k_{e f f} r} \int_{\theta=0}^{\pi} \frac{d P_{n}(\cos \theta)}{d \cos \theta} d \theta \\
\int_{\theta=0}^{\pi} E_{x}^{t} d \theta=E_{0} \sum_{n=1,3,5, \ldots}^{\infty} i^{n-1} \frac{2 n+1}{n(n+1)} \times \\
\times B_{n}^{t} \frac{\left[k_{m} r j_{n}\left(k_{m} r\right)\right]^{\prime}}{k_{m} r} \int_{\theta=0}^{\pi} \frac{d P_{n}(\cos \theta)}{d \cos \theta} d \theta .
\end{gathered}
$$

Видно, что суммы в этих интегралах содержат вклады электрических мультипольных возбуждений только нечетных порядков, т. е. электрического дипольного возбуждения $(n=1)$, электрического октупольного возбуждения $(n=3)$ и т. д.

Так как амплитуды мультипольных возбуждений $B_{n}^{r}$ и $B_{n}^{t}$ быстро убывают с увеличением порядка $n$, для упрощения дальнейших расчетов будем учитывать только дипольные возбуждения. Будем также считать, что размер области радиуса $R$, в которой проводится усреднение микроскопического поля, много меньше длины волны, т. е. $\left|k_{e f f} R\right| \ll$ $\ll 1$. Это неравенство является естественным условием для правомерности использования эффектив- 
ных электромагнитных параметров гетероструктуры при описании динамических процессов в ней. Оно позволяет в коэффициентах $B_{1}^{r}$ и $B_{1}^{t}$, а также в функции $\left[k_{e f f} r h_{n}^{(1)}\left(k_{e f f} r\right)\right]^{\prime}$ оставить только главные члены. Микроскопическое поле внутри металлической наночастцы будем описывать без использования квазистатического приближения, т. е. величину $\left|k_{m} a\right|$ не будем ограничивать никаким дополнительным неравенством, кроме естественного неравенства $a<R$.

Формула (203i) после подстановки в нее упрощен-

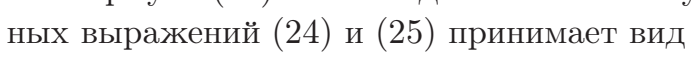

$$
\Delta \Phi_{m}=\frac{a^{3}}{R^{3}} \frac{\varepsilon_{m} J\left(k_{m} a\right)-\varepsilon_{e f f}}{\varepsilon_{m} J\left(k_{m} a\right)+2 \varepsilon_{e f f}} 2 \pi R^{2} \varepsilon_{e f f} E_{0},
$$

где функция $J(x)$ задана формулой

$$
J(x)=2 \frac{1-x \operatorname{ctg} x}{x^{2}+x \operatorname{ctg} x-1} .
$$

Аналогичным образом можно рассчитать вклад в скачок $\Delta \Phi_{d}$ от одиночной диэлектрической наночастицы, радиус $b$ которой можно считать сколь угодно малым. Этот вклад выражается похожей формулой

$$
\Delta \Phi_{d}=\frac{b^{3}}{R^{3}} \frac{\varepsilon_{d} J\left(k_{d} b\right)-\varepsilon_{e f f}}{\varepsilon_{d} J\left(k_{d} b\right)+2 \varepsilon_{e f f}} 2 \pi R^{2} \varepsilon_{e f f} E_{0} .
$$

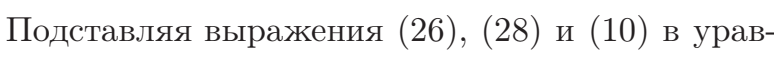

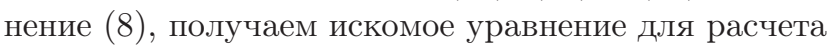
эффективной диэлектрической проницаемости гетероструктуры:

$$
c_{m} \frac{\varepsilon_{m} J\left(k_{m} a\right)-\varepsilon_{e f f}}{\varepsilon_{m} J\left(k_{m} a\right)+2 \varepsilon_{e f f}}+c_{d} \frac{\varepsilon_{d}-\varepsilon_{e f f}}{\varepsilon_{d}+2 \varepsilon_{e f f}}=0,
$$

где коэффициенты относительной объемной концентрации металла и диэлектрика заданы формулами $c_{m}=n_{m} a^{3} / R^{3}$ и $c_{d}=n_{d} b^{3} / R^{3}$ и связаны условием $c_{m}+c_{d}=1$. Здесь учтено, что функция $J(x)$ при $|x| \ll 1$ является константой, равной единице.

В результате из выражения (129.') получаем уравнение, которое является квадратичным относительно искомой величины $\varepsilon_{e f f}$, а потому имеет два решения:

$$
\varepsilon_{e f f}=\frac{H_{\varepsilon} \pm \sqrt{H_{\varepsilon}^{2}+8 \varepsilon_{m} \varepsilon_{d} J\left(k_{m} a\right)}}{4},
$$

где введено обозначение

$$
H_{\varepsilon}=\left(2-3 c_{m}\right) \varepsilon_{d}-\left(1-3 c_{m}\right) \varepsilon_{m} J\left(k_{m} a\right) .
$$

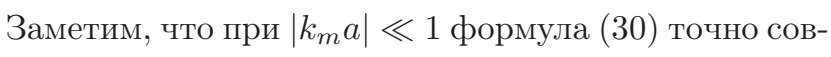


рассматривать решение только со знаком плюс.
В монографии [1를, а также в работе [른] при выборе решения уравнения Бруггемана предлагается оставлять только знак плюс перед квадратным корнем. Однако, учитывая тот факт, что диэлектрическая проницаемость $\varepsilon_{m}$ металлических частиц комплексна, такой выбор решения для эффективной диэлектрической проницаемости $\varepsilon_{e f f}$ не всегда корректен.

Действительно, как известно, положительный знак мнимой части $\varepsilon_{e f f}$ говорит о наличии диэлектрических или омических потерь в среде, определяющих затухание электромагнитной волны, а отрицательный знак противоречит физике - он говорит об усилении электромагнитной волны, т. е. о генерации энергии в структуре. Поэтому перед квадратным корнем следует выбирать такой знак, чтобы мнимая часть $\varepsilon_{e f f}$ всегда оставалась только положительной. Правильный выбор знака для решения уравнения $\left(\overline{2} \overline{9}_{1}^{\prime}\right)$ автоматически обеспечивается, если формулу (30.0

$$
\varepsilon_{e f f}=\frac{H_{\varepsilon}+i \sqrt{-H_{\varepsilon}^{2}-8 \varepsilon_{m} \varepsilon_{d} J\left(k_{m} a\right)}}{4} .
$$

Важно заметить, что знак Re $\varepsilon_{e f f}$ может быть любым.

Проводя аналогичные выкладки для магнитных полей $\mathbf{H}^{r}$ и $\mathbf{H}^{t}$, заданных формулами (11 $\left.\overline{3}_{1}^{\prime}\right)$ и (11 $\left.\overline{7}_{-1}^{\prime}\right)$, можно рассчитать эффективную магнитную проницаемость диэлектрической среды, содержащей металлические наночастицы. При этом несложно показать, что вклад в $\mu_{e f f}$ дают только магнитные мультипольные колебания нечетного порядка. Однако для простоты в расчете будем учитывать только магнитодипольные колебания. В результате получаем формулу для расчета эффективной относительной магнитной проницаемости диэлектрической среды с металлическими наночастицами:

$$
\mu_{e f f}=\frac{H_{\mu}+i \sqrt{-H_{\mu}^{2}-8 \mu_{m} \mu_{d} J\left(k_{m} a\right)}}{4},
$$

где

$$
H_{\mu}=\left(2-3 c_{m}\right) \mu_{d}-\left(1-3 c_{m}\right) \mu_{m} J\left(k_{m} a\right) .
$$

При $\mu_{m}=\mu_{d}$ и $\left|k_{m} a\right| \ll 1$ формула (323iz) принимает простой вид:

$$
\mu_{e f f}=\mu_{d}\left(1+\frac{c_{m}}{10} \frac{\omega^{2}}{c^{2}} a^{2} \varepsilon_{m}\right) .
$$

Заметим, что даже при $\mu_{m}=\mu_{d}=1$ эффективная магнитная проницаемость $\mu_{e f f}$ гетероструктуры не будет равняться единице, если $k_{m} a \neq 0$, что ранее отмечалось в работе [표요. 
Проведенный расчет показывает, что электрические мультипольные колебания любого порядка в наночастицах не вносят вклада в эффективную магнитную проницаемость гетероструктуры, также как и магнитные мультипольные колебания любого порядка не вносят вклада в эффективную диэлектрическую проницаемость рассматриваемой среды.

\section{4. РЕЗУЛЬТАТЫ ИССЛЕДОВАНИЙ И ИХ АНАЛИЗ}

Было отмечено, что отличие полученных в настоящей работе формул от формул Максвелла Гарнетта и Бруггемана заключается в том, что наши формулы отражают зависимости эффективных электромагнитных параметров гетероструктуры от размеров наночастиц, содержащихся в диэлектрической матрице. Важно также отметить, что при радиусе наночастиц $a<5$ нм и их концентрации $c_{m}<$ $<10^{-6}$ частотные зависимости компонент эффективной комплексной диэлектрической проницаемости $\varepsilon_{e f f}=\varepsilon^{\prime}+i \varepsilon^{\prime \prime}$, построенные как по формулам Максвелла Гарнетта и Бруггемана, так и по нашим формулам, практически неразличимы. Однако при концентрации $c_{m}>10^{-5}$ зависимости $\varepsilon^{\prime}(f)$ и $\varepsilon^{\prime \prime}(f)$, построенные по формуле Максвелла Гарнетта, значительно отличаются от соответствующих зависимостей, построенных как по формуле Бруггемана, так и по полученной нами формуле.

На рис. 'I' для водной суспензии частиц серебра с концентрацией $c_{m}=10^{-3}$ приведены частотные зависимости компонент эффективной комплексной диэлектрической проницаемости, рассчитанные по

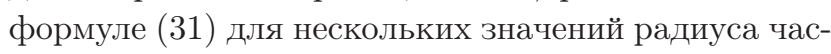
тиц, а также зависимости, рассчитанные по фор-

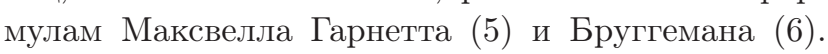
В расчетах диэлектрическая проницаемость метал-

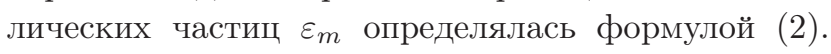
При этом значения входящих в нее параметров взяты из работы [īi]|: плазменная частота $\omega_{p} / 2 \pi=$ $=1600$ ТГц, частота релаксации носителей заряда $\gamma / 2 \pi=20$ ТГц, оптическая диэлектрическая проницаемость металла $\varepsilon_{\infty}=1$. Диэлектрическая проницаемость воды (диэлектрической матрицы исследуемой гетероструктуры), согласно данным из [292in', равна $\varepsilon_{d}=1.788$. Все эти параметры использовались нами и в дальнейших исследованиях.

Зависимости на рис. '1' построены в узком частотном диапазоне в области резонансной частоты $f_{1}$ дипольных $(n=1)$ колебаний частиц. Видно, что резонансные кривые, рассчитанные по форму-
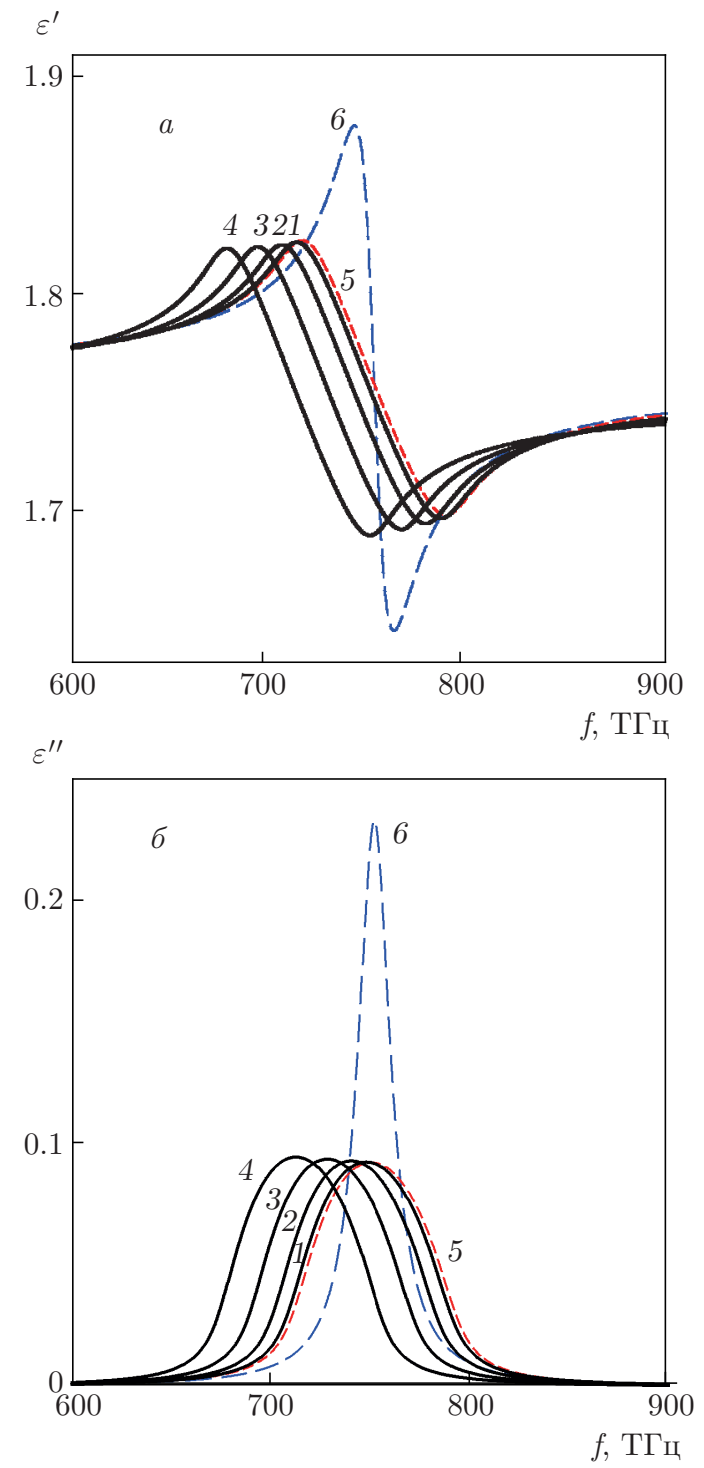

Рис. 1. (В цвете онлайн) Частотные зависимости действительной $(a)$ и мнимой (б) частей эффективной диэлектрической проницаемости водной суспензии наночастиц серебра при $c_{m}=1 \cdot 10^{-3}$, рассчитанные по формуле (힌) для $a=10$ нм (1), 20 нм (2), 30 нм (3), 40 нм (4), а также по формулам Бруггемана (5) и Максвелла Гарнетта (6)

лам Максвелла Гарнетта и Бруггемана, значительно различаются. В частности, максимумы мнимых компонент диэлектрических проницаемостей, $\varepsilon_{1}^{\prime \prime}$, характеризующих поглощение в гетероструктуре, и ширины резонансных линий, $\Delta f_{1}$, измеренные по среднему уровню от максимальных величин, различаются больше, чем в два раза, хотя резонансные частоты $f_{1}$ совпадают. На рис. 1'1; также видно, что

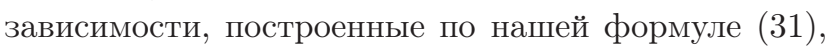
в отличие от формул Максвелла Гарнетта и Бругге- 
мана, показывают существенное понижение частоты плазмонного резонанса $f_{1}$ при увеличении размера металлических наночастиц $a>10$ нм. Однако при $a<5$ нм резонансные кривые, рассчитанные по

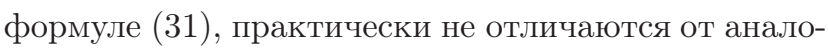
гичных кривых, рассчитанных по формуле Бруггемана $\left(\overline{6_{1}^{\prime}}\right)$.

Очевидно, что если в гетероструктуре не содержатся магнитные частицы, то ее эффективная магнитная проницаемость $\mu_{e f f}$ близка к единице. Поэтому магнитные свойства рассматриваемой водной суспензии серебра удобнее описывать с помощью эффективной относительной магнитной восприимчивости $\chi_{e f f}=\mu_{e f f}-1$. На рис. $\overline{2}_{1}^{1}$ в широком диапазоне частот построены зависимости $\chi_{e f f}=\chi^{\prime}+i \chi^{\prime \prime}$, рассчитанные по формуле (32i-') также для нескольких размеров наночастиц серебра при их концентрации в суспензии $c_{m}=1 \cdot 10^{-3}$. При этом считалось, что $\mu_{m}=\mu_{d}=1$.

Отметим, что на действительной части магнитной восприимчивости $\chi^{\prime}(f)$ гетероструктуры в рассматриваемом диапазоне частот не проявляется магнитодипольный резонанс, так как его частота, в отличие от электродипольного резонанса, существенно выше этого диапазона. Однако на зависимости $\chi^{\prime \prime}(f)$ в дальней инфракрасной области наблюдается ярко выраженный максимум, частота которого $f_{\max }$, как показывают исследования, практически не зависит от размера частиц в суспензии, если их радиус $a<30$ нм. На вставке к рис. 룰 приведен фрагмент области максимума $\chi^{\prime \prime}(f)$, на котором хорошо видно, что $f_{\max } \approx 20$ ТГц, но следует заметить, что при увеличении размеров наночастиц частота максимума монотонно понижается.

Для объяснения природы существования нерезонансного максимума на частотной зависимости мнимой компоненты магнитной восприимчивости суспензии подставим комплексную диэлектрическую проницаемость $\varepsilon_{m}(\omega)$ материала наночастицы (см.

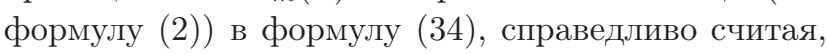
что в выбранной области частот размеры частиц много меньше длины волны, $\left|k_{m} a\right| \ll 1$. Выделим затем мнимую часть в полученном выражении:

$$
\chi^{\prime \prime}=\frac{c_{m} \gamma}{10} \frac{\omega_{p}^{2}}{c^{2}} a^{2} \frac{\omega^{2}}{\omega^{2}+\gamma^{2}} .
$$

Отсюда видно, что максимум мнимой части эффективной магнитной восприимчивости располагается точно на частоте релаксации носителей заряда $\omega_{\max }=\gamma$. Этот факт показывает возможность экспериментального определения частоты релаксации носителей заряда наночастиц в рассматриваемой ге-
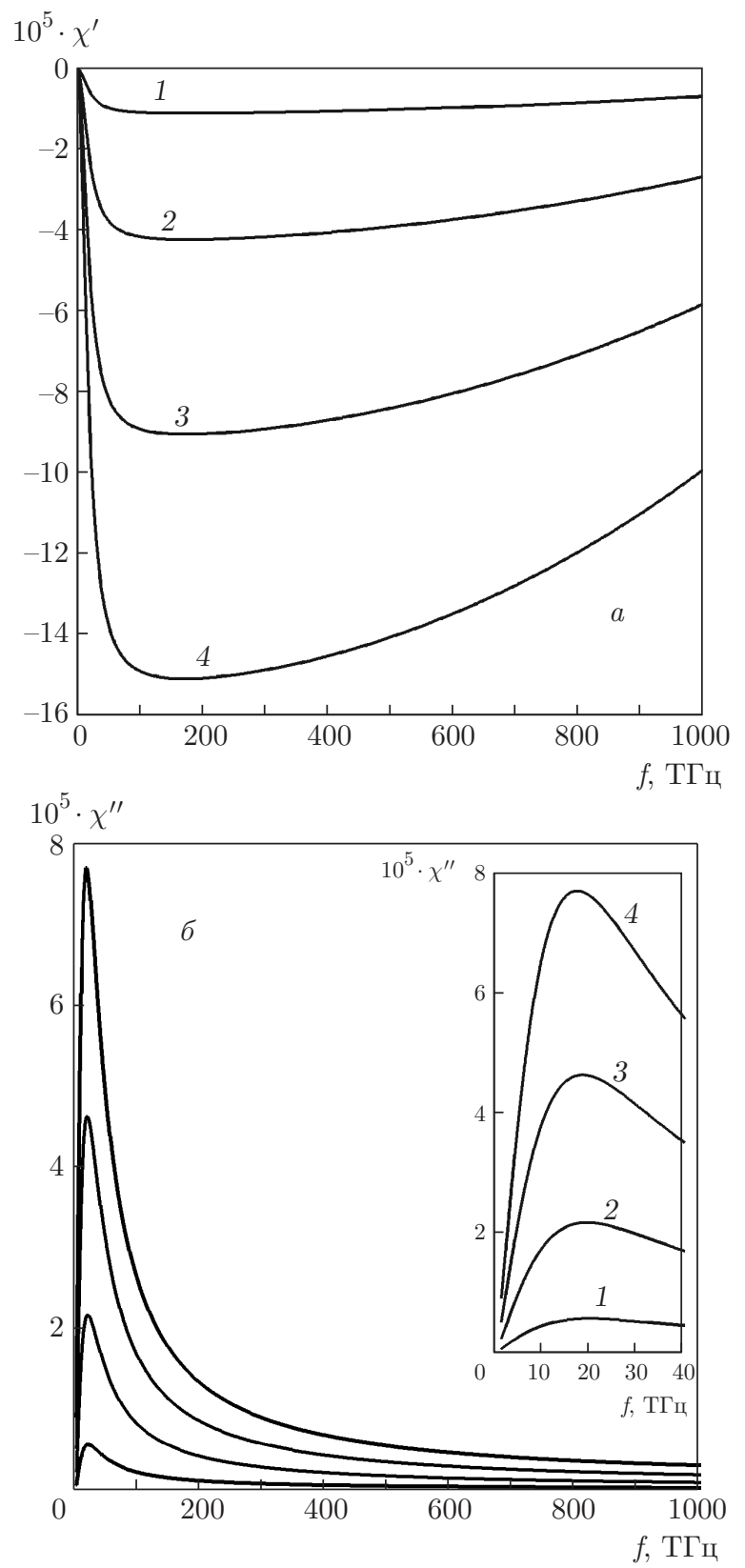

Рис. 2. (В цвете онлайн) Частотные зависимости действительной $(a)$ и мнимой (б) частей эффективной относительной магнитной восприимчивости водной суспензии наночастиц серебра при $c_{m}=1 \cdot 10^{-3}$, рассчитанные по формуле (믄) : $1-a=10$ нм; $2-a=20$ нм; $3-a=30$ нм; $4-$ 7 $a=40 \mathrm{Hм}$

тероструктуре. Для этого необходимо лишь измерить частоту максимума затухания электромагнитных волн, которая, очевидно, совпадает с частотой максимума на зависимости $\chi^{\prime \prime}(f)$.

На рис. "2 также видно, что действительная часть $\chi^{\prime}$ всегда отрицательна. Это значит, что гетеро- 
структуры, содержащие немагнитные металлические частицы в диэлектрической матрице, проявляют свойства диамагнетиков с величиной эффективной магнитной восприимчивости порядка $10^{-5}$ для рассмотренной концентрации и размеров наночастиц. Однако с увеличением радиуса $a$ наночастиц, а также с увеличением их объемной концентрации $c_{m}$ в гетероструктуре эффективная магнитная восприимчивость $\chi_{\text {eff }}$ может возрасти по абсолютной величине на порядки. Например, при $a=100$ нм и $c_{m}=0.1$ мнимая компонента восприимчивости, измеренная на частоте релаксации носителей заряда $(\gamma / 2 \pi=20$ ТГц $)$, возрастает до $\chi^{\prime \prime}=2.2 \cdot 10^{-2}$, а действительная компонента на частоте своего минимума $f_{\min }=153$ ТГц возрастает до $\chi^{\prime}=-5.4 \cdot 10^{-2}$. Отметим, что в статическом пределе $\chi_{e f f}$ обращается в нуль, что хорошо видно на рис. $\underline{12}_{-\mathbf{r}}^{\prime}$

На рис. $\overline{3}-1$ фективной комплексной диэлектрической проницаемости $\varepsilon_{e f f}$, рассчитанные при концентрации частиц серебра в водной суспензии $c_{m}=1 \cdot 10^{-2}$ для нескольких размеров наночастиц (кривые $1-3)$. При сравнении графиков на рис. $\overline{1} 1$ и рис. $\overline{1}$ видно что, увеличение концентрации $c_{m}$ в десять раз приводит к увеличению на порядок максимума мнимой компоненты эффективной диэлектрической проницаемости $\varepsilon_{1}^{\prime \prime}$, рассчитанной по формуле Максвелла Гарнетта. Однако аналогичная величина, рассчитанная по формуле Бруггемана, увеличилась всего примерно в 3.5 раза. При этом различие между этими двумя максимальными значениями $\varepsilon_{1}^{\prime \prime}$ увеличилось с двух раз (при $c_{m}=1 \cdot 10^{-3}$ ) до семи (при $c_{m}=1 \cdot 10^{-2}$ ).

На рис. "4 представлены зависимости резонансной частоты $f_{1}$, а также максимального (резонансного) значения мнимой компоненты эффективной диэлектрической проницаемости $\varepsilon_{1}^{\prime \prime}$ от радиуса $a$ на-

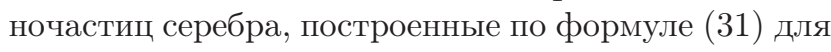
нескольких концентраций $c_{m}$ (кривые 1-3). Здесь же прямыми линиями 4, 5 представлены значения соответствующих величин, рассчитанных по формулам Бруггемана только для двух концентраций, чтобы не загромождать рисунок, а прямыми линиями 6,7 по формулам Максвелла Гарнетта, при выводе которых размеры частиц считаются бесконечно малыми.

Видно, что резонансные частоты, вычисленные

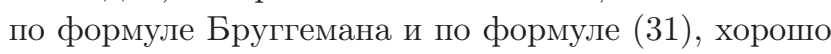
совпадают для любых концентраций частиц в суспензии, но только при очень малых размерах частиц. Резонансная частота, вычисленная по формуле Максвелла Гарнетта, согласуется с этими расчетами также при очень малых размерах наночастиц, но только при очень малых их концентрациях $c_{m}$ в
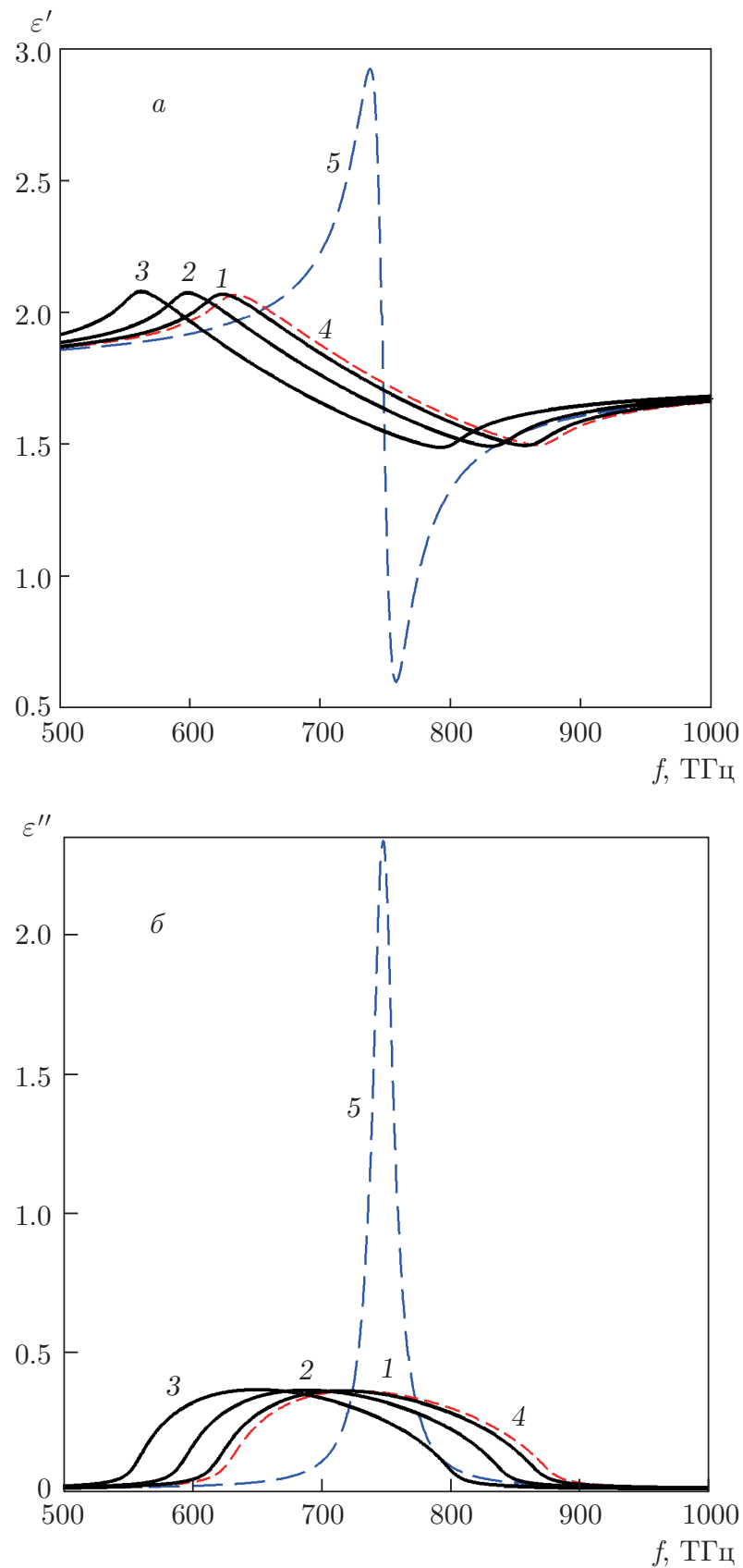

Рис. 3. (В цвете онлайн) Частотные зависимости действительной (a) и мнимой (б) частей эффективной диэлектрической проницаемости водной суспензии наночастиц серебра при $c_{m}=1 \cdot 10^{-2}$, рассчитанные по формуле ('3-1') для $a=20$ нм (1), 40 нм (2), 60 нм (3), а также по формулам Бруггемана (4) и Максвелла Гарнетта (5)

суспензии. Как и следовало ожидать, резонансная

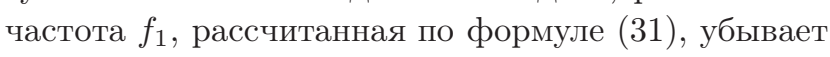
не только с увеличением радиуса $a$, но и с увеличением концентрации частиц в суспензии. При этом резонансная величина $\varepsilon_{1}^{\prime \prime}$ мнимой части диэлектри- 



Pис. 4. (В цвете онлайн) Зависимости резонансной частоты $f_{1}(a)$ и резонансного значения мнимой части эффективной диэлектрической проницаемости $\varepsilon_{1}^{\prime \prime}$ (б) от радиуса наночастиц серебра, вычисленные по формуле (131) для $c_{m}=0.001$ (1), 0.01 (2), 0.1 (3), а также по формуле Бруггемана для $c_{m}=0.001(4), 0.1$ (5) и формуле Максвелла Гарнетта для $c_{m}=0.001(6,7)$

ческой проницаемости слабо увеличивается с увеличением радиуса $a$, но быстро растет с увеличением концентрации частиц в суспензии. При концентрации наночастиц в суспензии $c_{m}=0.1$ максимальное значение мнимой части диэлектрической проницае- мости, вычисленное по формуле Максвелла Гарнетта, слишком большое, $\varepsilon_{1}^{\prime \prime}=23.86$, поэтому оно не приведено на рис. $\overline{4} \underset{1}{1}$

Завышение эффективной диэлектрической проницаемости, имеющее место при расчете по формуле Максвелла Гарнетта и увеличивающееся с ростом концентрации частиц в гетероструктуре, объясняется следующим. Во-первых, в квазистатическом расчете Максвелла Гарнетта при вычислении диэлектрической поляризации $P$ металлических наночастиц (см. формулу $(\overline{7} \mathbf{7}))$ используется модель, в которой реальная частица заданных размеров, обладающая дипольным моментом, заменяется точечной частицей с тем же дипольным моментом. При этом электрическое поле внутри частицы считается таким же, как и поле в окружающей диэлектрической матрице, т. е. пренебрегается деполяризующим полем внутри частицы. Во-вторых, в расчете Максвелла Гарнетта не учитывается взаимодействие частиц.

Очевидно, что столь грубые приближения могут быть правомерными только при очень малых концентрациях частиц в гетероструктуре. Исследования показали, что уже при $c_{m}>10^{-6}$ такие приближения дают заметную погрешность, быстро нарастающую с увеличением $c_{m}$.

Это подтверждают кривые частотной зависимости $\varepsilon^{\prime \prime}(f)$, построенные для нескольких концентраций $c_{m}$ на рис. п्ञ пороле Максвелла Гарнетта (кривая 1) и по формуле Бруггемана (кривые 2-4). Для наглядности все кривые нормированы на соответствующую концентрацию $c_{m}$. Отметим, что зависимости, построенные по формуле Максвелла Гарнетта для концентраций $c_{m}=1 \cdot 10^{-5}, 1 \cdot 10^{-4}, 1 \cdot 10^{-3}$ показаны одной кривой 1 , так как они практически неразличимы.

В квазистатическом расчете Бруггемана поле внутри частицы считается однородным в предположении, что ее размеры много меньше длины волны, однако деполяризующее поле учитывается. Такое приближение, очевидно, также требует малости размеров частиц. Достоинством модели Бруггемана, как уже отмечалось, является учет взаимодействия выделенной частицы с другими наночастицами гетероструктуры в приближении усредненного поля. Учет взаимодействия частиц проявляется в виде сильного уширения резонансной кривой $\varepsilon^{\prime \prime}(f)$ при увеличении их концентрации $c_{m}$ в гетероструктуре (см. рис. 高). Важной характеристикой любого резонанса является его добротность, которую можно рассчитать по традиционной формуле $Q_{1}=f_{1} / \Delta f_{1}$. 




Рис. 5. (В цвете онлайн) Частотные зависимости мнимой части эффективной диэлектрической проницаемости водной суспензии наночастиц серебра, вычисленные по формуле Максвелла Гарнетта для $c_{m}=1 \cdot 10^{-5}-1 \cdot 10^{-3}(1)$ и по формуле Бруггемана для $c_{m}=1 \cdot 10^{-5}(2), c_{m}=1 \cdot 10^{-4}(3)$,

$$
c_{m}=1 \cdot 10^{-3}(4)
$$

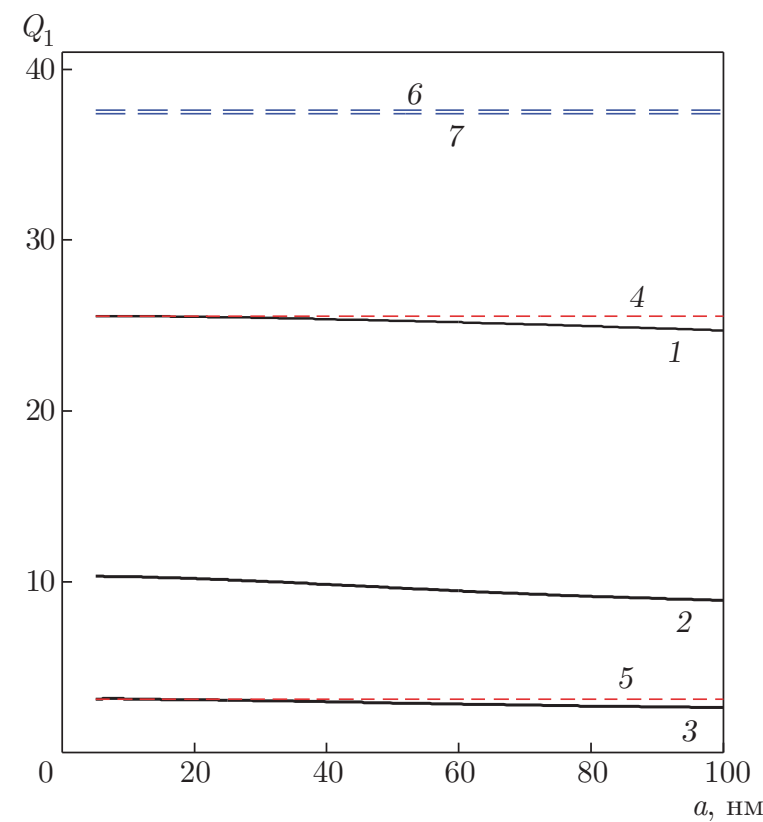

Рис. 6. (В цвете онлайн) Зависимости добротности электродипольного плазмонного резонанса в водной суспензии наночастиц серебра от их радиуса, вычисленные в настоящей работе для $c_{m}=0.0001$ (1), 0.001 (2), 0.01 (3), а также по формуле Бруггемана для $c_{m}=0.0001$ (4), 0.01 (5) и по формуле Максвелла Гарнетта для $c_{m}=0.0001$ (6), $0.01(7)$
На рис. '行 сплошными линиями 1-3 на основе нашего расчета построены зависимости добротности $Q_{1}$ плазмонного электродипольного резонанса от радиуса $a$ частиц серебра в водных суспензиях с различной концентрацией наночастиц. Видно, что добротность монотонно убывает с увеличением радиуса частиц, но гораздо быстрее уменьшается с ростом концентрации наночастиц в суспензии. Здесь же для двух концентраций наночастиц в суспензии прямыми линиями 4 и 5 показаны значения $Q_{1}$, получающиеся по расчету Бруггемана, а прямыми линиями 6 и 7 - по расчету Максвелла Гарнетта. Видно, что при малых размерах частиц добротность плазмонного электродипольного резонанса по расчету Бруггемана хорошо совпадает с нашим расчетом. Однако добротность $Q_{1}$, получаемая по расчету Максвелла Гарнетта, во-первых, значительно завышена, а вовторых, она почти не зависит от концентрации наночастиц в суспензии.

\section{5. ЗАКЛЮЧЕНИЕ}

Представленный электродинамический расчет частотных зависимостей эффективной комплексной диэлектрической проницаемости и эффективной комплексной магнитной восприимчивости гетероструктуры, состоящей из диэлектрической матрицы, содержащей металлические наночастицы сферической формы, учитывает размеры наночастиц. В отличие от подходов Бруггемана и Максвелла Гарнетта, использующих в расчетах квазистатическое приближение, в котором размеры частиц считаются много меньше длины волны в металле, рассчитано неоднородное распределение электромагнитных полей внутри металлических наночастиц. Установлено, что электрические мультипольные колебания любого порядка в наночастицах не вносят вклада в эффективную магнитную проницаемость гетероструктуры, так же как и магнитные мультипольные колебания любого порядка не вносят вклада в эффективную диэлектрическую проницаемость рассматриваемой среды. При этом вклад в эффективную комплексную диэлектрическую проницаемость $\varepsilon_{\text {eff }}$ могут вносить только электрические мультипольные возбуждения нечетных порядков, т. е. дипольные, октупольные и т. д., так же как и вклад в эффективную комплексную магнитную проницаемость $\mu_{e f f}$ могут вносить только магнитные мультипольные возбуждения нечетных порядков. 
Показано, что частота плазмонного электродипольного резонанса понижается как с увеличением размера частиц, так и с увеличением их концентрации в гетероструктуре. Эти факты подтверждаются экспериментальными исследованиями водных

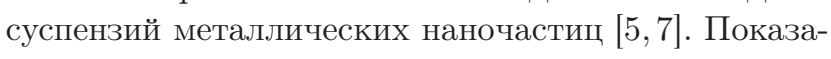
но также, что диэлектрическая среда, содержащая немагнитные металлические наночастицы, проявляет диамагнитные свойства, что предполагает необходимость проведения экспериментальных исследований в этой области. Важным результатом работы является установленный факт, что частота максимума на частотной зависимости мнимой части магнитной восприимчивости совпадает с частотой релаксации носителей заряда. Благодаря этому факту появляется возможность экспериментального измерения частоты релаксации по частоте максимума поглощения электромагнитного излучения гетероструктурой при возбуждении в ней магнитодипольных колебаний.

Рассчитанные спектры действительной и мнимой компонент диэлектрической проницаемости гетероструктуры с размерами металлических частиц меньше 10 нм хорошо согласуются с расчетами Бруггемана, однако с расчетами Максвелла Гарнетта согласие наблюдается только при очень низких концентрациях наночастиц, меньших $10^{-6}$. Это объясняется слишком грубыми приближениями, используемыми в расчете Максвелла Гарнетта, в частности, в нем не учитывается взаимодействие частиц гетероструктуры, которые учтены в расчете Бруггемана в приближении среднего поля. Кроме того, в расчете Максвелла Гарнетта не учитывается деполяризующее поле, так как в нем частица конечных размеров заменяется точечным диполем. Тем не менее многие авторы в своих исследованиях используют подход Максвелла Гарнетта [1 1 ; и используют его формулу в своих исследованиях даже при высоких концентрациях частиц в гетероструктуре до $c_{m}=0.2$ [3] $\left.\underline{1}_{1}^{\prime}\right]$.

Работа выполнена при поддержке Министерства образования и науки РФ (соглашение № 14.575.21.0142, уникальный идентификатор проекта RFMEFI57517X0142).

\section{ЛИТЕРАТУРА}

1. M. Mishra and P. Chauhan, J. Nanomed. Res. 2, 00039 (2015).
2. H. Malekzad, P. S. Zangabad, H. Mirshekari et al., Nanotechnol. Rev. 6, 301 (2017).

3. M. R. Díaz and P. E. Vivas-Mejia, Pharmaceuticals 6, 1361 (2013).

4. А. А. Заболотский, ЖЭЭФ 146, 700 (2014).

5. W. Jacak, J. Krasnyj, J. Jacak et al., J. Appl. Phys. 107, 124317 (2010).

6. B. A. Belyaev and V. V. Tyurnev, Microw. Opt. Tech. Lett. 58, 1883 (2016).

7. S. J. Oldenburg, https://www.sigmaaldrich.com/ technical-documents/articles/materials-science/ nanomaterials/silver-nanoparticles.html (2017).

8. G. Mie, Ann. Phys. 25, 4, 377 (1908).

9. Дж. А. Стрэттон, Теория электромагнетизма, ОГИЗ, Москва (1948), гл. ІХ.

10. F. Frezza, F. Mangini, and N. Tedeschi, J. Opt. Soc. Amer. A 35, 163 (2018).

11. В. А. Астапенко, С. Ю. Свита, ЖЭТФ 148, 444 (2015).

12. T. C. Choy, Effective Medium Theory: Principles and Applications, Oxford Univ. Press, Oxford (2016).

13. W. T. Doyle, Phys. Rev. B 39, 9852 (1989).

14. D. J. Bergman, Sol. St. Phys. 56, 147 (1992).

15. V. N. Pustovit, T. V. Shahbazyan, and L. G. Grechko, Eur. Phys. J. B 69, 369 (2009).

16. M. Sancho and G. Martinez, J. Electrostat. 22, 319 (1989).

17. J. C. Maxwell Garnett, Phil. Trans. Roy. Soc. London A 203, 385 (1904).

18. A. Sihvola, J. Electromag. Waves Appl. 15, 715 (2001).

19. Л. А. Головань, В. Ю. Тимошенко, П. К. Кашкаров, УФН 177, 6, 619 (2007).

20. M. Scheller, C. Jansen, and M. Koch, in Recent Optical and Photonic Technologies, ed. by K. Y. Kim, Intech, Croatia, Vukovar (2010), p. 231.

21. D. A. G. Bruggeman, Ann. der Phys. Ser. 5, 24, 636 (1935).

22. R. Ruppin, Opt. Comm. 182, 273 (2000).

23. C. A. Grimes and D. M. Grimes, Phys. Rev. B 43, 10780 (1991).

24. A. Lakhtakia, Optik 91, 134 (1992).

25. C. A. Foss, G. L. Hornyak, J. A. Stockert et al., J. Phys. Chem. 98, 2963 (1994). 
26. N. A. F. Al-Rawashdeh, M. L. Sandrock, C. J. Seugling et al., J. Phys. Chem. B 102, 361 (1998).

27. D. Zhang, E. Cherkaev, and M. P. Lamoureux, Appl. Math. Comp. 217, 7092 (2011).

28. C. F. Bohren, J. Atmosph. Sci. 43, 468 (1986).

29. S. Kedenburg, M. Vieweg, and H. Giessen, Opt. Mater. Expr. 2, 1588 (2012).
30. P. Mallet, C. A. Guérin, and A. Sentenac, Phys. Rev. B 72, 014205 (2005).

31. И. В. Тимофеев, Автореферат дисс. на соискание уч. ст. доктора физ.-мат. наук. ИФ СО РАН, Красноярск (2017), http://kirensky.ru/zdoc/ ref_timofeev.pdf. 